NASA/CR-201744

ICASE Report No. 97-49

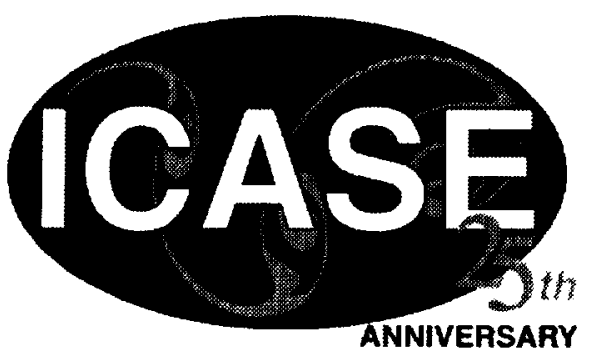

\title{
The Analysis and Construction of Perfectly Matched Layers for Linearized Euler Equations
}

\author{
J. S. Hesthaven \\ Brown University
}

Institute for Computer Applications in Science and Engineering NASA Langley Research Center

Hampton, VA

Operated by Universities Space Research Association

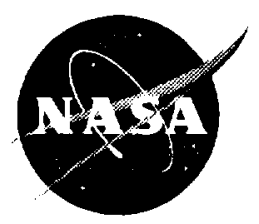

National Aeronautics and Space Administration 
Available from the following:

NASA Center for AeroSpace Information (CASI) 800 Elkridge Landing Road

Linthicum Heights, MD 21090-2934

(301) 621-0390
National Technical Information Service (NTIS)

5285 Port Royal Road

Springfield, VA 22161-2171

(703) 487-4650 


\title{
ON THE ANALYSIS AND CONSTRUCTION OF PERFECTLY MATCHED LAYERS FOR THE LINEARIZED EULER EQUATIONS
}

\author{
J. S. HESTHAVEN *
}

\begin{abstract}
We present a detailed analysis of a recently proposed perfectly matched layer (PML) method for the absorption of acoustic waves. The split set of equations is shown to be only weakly well-posed, and ill-posed under small, low order, perturbations. This analysis provides an explanation for the stability problems associated with the spilt field formulation and illustrates why applying a filter has a stabilizing effect.

Utilizing recent results obtained within the context of electromagnetics, we develop strongly well-posed absorbing layers for the linearized Euler equations. The schemes are shown to be perfectly absorbing independent of frequency and the angle of incidence of the wave in the case of a quiescent mean flow. In the general case of a convecting mean flow, a number of techniques are combined to obtain an absorbing layer exhibiting PML-like behavior. The efficiency of the proposed absorbing layer is illustrated through the computation of benchmark problems in aero-acoustics.
\end{abstract}

Key words. acoustics, absorbing boundary conditions, hyperbolic systems

Subject classification. Applied and Numerical Mathematics, Fluid Mechanics

1. Introduction. When addressing wave-dominated problems, as found in aero-acoustics or electromagnetics, one is often facing the problem of how to accurately obtain infinite domain solutions using a finite computational domain. The truncation of the computational domain must be done in a way that avoids, at least approximately, the excitation of reflected waves which might otherwise contaminate the computational domain and falsify the solution.

The issue of how to properly devise such boundary conditions at an artificial computational boundary has received much attention in past. The use of characteristic boundary conditions [1] is appealing due to its simplicity, but is only accurate for close to perpendicular incident of waves. More elaborate schemes involve radiation boundary conditions based on localization of the Dirichlet-to-Neumann map [2, 3] or an asymptotic expansion of the far-field solution [4]. A fairly recent review of such methods can be found in [5]. Alternatives to such schemes involve the introduction of buffer or sponge layers in which the waves are either damped [6], accelerated to supersonic conditions [7], decelerated [8] or attenuated by combinations thereof [9]. The construction of such schemes are in most cases based on physical arguments with little theoretical evidence of their, often quite remarkable, performance.

In the context of electromagnetics, Berenger [10] recently proposed a novel way by which to derive the sought after absorbing boundary conditions. By splitting Maxwell's equations in an unphysical manner, additional degrees of freedom are introduced thus allowing for the construction of absorbing layers. These layers have the remarkable property that they maintain their absorbing properties irrespective of the frequency and angle of propagation of the incident wave, i.e. this approach appears to provide an optimal absorbing boundary condition. These layers, called Perfectly Matched Layers (PML), are now under intensive research.

*Division of Applied Mathematics, Brown University, Box F, Providence, RI 02912, e-mail: jansh@cfm.brown.edu. This research was partially supported by the National Aeronautics and Space Administration under NASA Contract Nos. NAS197046 and NAS1-19480 while the author was in residence at the Institute for Computer Applications in Science and Engineering(ICASE), M/S 403, NASA Langley Research Center, Hampton, VA, 23681-0001 
While the PML scheme has been applied successfully during the last years, it was recently proven [11] that the splitting of Maxwell's equations makes the resulting set of equations only weakly well-posed and ill-posed under arbitrary low order perturbations, i.e. the numerical schemes resulting from these equations can be expected to be unconditionally unstable, an example of such being provided in [11]. This realization has focused the attention towards alternative well-posed formulations of the electromagnetic PML methods and several such schemes have been proposed in recent years, see. e.g. $[12,13,14,15,16,17]$. Hence, although the original approach for the construction of PML schemes have proven erroneous, the general approach have proven extremely fruitful and allowed for the computation of problems in electromagnetics of unsurpassed accuracy.

Inspired by the success of the PML methods for Maxwell's equations, Hu [18] recently proposed a PML method for the equations of acoustics by taking an approach very similar to the one originally developed for Maxwell's equations, i.e. by splitting the equations of acoustics in an unphysical manner. However, contrary to most work within the community of electromagnetics, $\mathrm{Hu}[18]$ reported the need for using a low pass filter inside the absorbing layers to maintain stability of the scheme. A similar observation was made in [19] where no filter is applied and the numerical solutions are found to exhibit exponential growth. This points to an inherent instability of the scheme for which a partial explanation, in terms of loss of strong well-posedness in certain special cases, is providedin [19].

In the present work we provide a complete analysis of the split PML scheme of [18], confirming the speculations put forward in [19] in a more general context. Indeed, the scheme of [18] is found to be only weakly well-posed in the two-dimensional case and ill-posed under low order perturbations. We proceed by presenting a well-posed PML scheme for the quiescent equations of acoustics, and a well-posed absorbing layer exhibiting PML-like behavior for the general convective case.

The remainder of this paper is organized as follows. In Sec. 2 we introduce the equations of acoustics as obtained from the linearized Euler equations. Section 3 contains the first part of the paper in which we present an analysis of the PML method recently proposed in [18] and provide an explanation for the problems of stability reported in $[18,19]$. This leads to Sec. 4 , where we present an alternative to the unstable PML scheme. For the case of a quiescent mean flow we construct a well behaved PML method and illustrate its performance through numerical experiments. For the general case of a convective mean flow, we propose to apply a combination of techniques to arrive at absorbing layers with PML-like behavior and support the reasoning by numerical studies. Section 5 contains a few concluding remarks.

2. The Equations of Acoustics. We shall consider the two-dimensional compressible Euler equations, linearized around a uniform parallel mean flow of the form $\left(\rho_{0}, u_{0}, 0, p_{0}\right)$. Choosing $v_{0}=0$ does not introduce any restrictions on the analysis as the general situation may always be rotated to arrive at this particular case. Within this scenario, the equations take the form

$$
\frac{\partial \boldsymbol{q}}{\partial t}+\mathrm{A} \frac{\partial \boldsymbol{q}}{\partial x}+\mathrm{B} \frac{\partial \boldsymbol{q}}{\partial y}=\mathbf{0}
$$

where the state vector, $\boldsymbol{q}$, and the constant matrices, $\mathrm{A}$ and $\mathrm{B}$, are given as

$$
\boldsymbol{q}=\left[\begin{array}{c}
\rho \\
u \\
v \\
p
\end{array}\right], \quad \mathrm{A}=\left[\begin{array}{cccc}
M & 1 & 0 & 0 \\
0 & M & 0 & 1 \\
0 & 0 & M & 0 \\
0 & 1 & 0 & M
\end{array}\right], \mathrm{B}=\left[\begin{array}{llll}
0 & 0 & 1 & 0 \\
0 & 0 & 0 & 0 \\
0 & 0 & 0 & 1 \\
0 & 0 & 1 & 0
\end{array}\right]
$$


These equations are recovered from the Euler equations by linearizing around the uniform mean state, $\left(\rho_{0}, u_{0}, 0, p_{0}\right)$, and introducing the normalization

$$
t=\frac{t c_{0}}{L}, x=\frac{x}{L}, y=\frac{y}{L}, \boldsymbol{q}=\left[\frac{\rho}{\rho_{0}}, \frac{u}{c_{0}}, \frac{v}{c_{0}}, \frac{p}{\rho_{0} c_{0}^{2}}\right]^{T},
$$

where $L$ represents a characteristic length while $c_{0}=\sqrt{\gamma p_{0} / \rho_{0}}$ refers to the sound speed of the mean flow. In this context, Eqs.(1)-(2), describes the propagation and interaction of waves in a parallel uniform flow with a Mach number, $M=u_{0} / c_{0}$.

A deeper understanding of the underlying properties, physical as well as mathematical, of Eqs.(1)-(2) can be gained by introducing the similarity transform

$$
\mathbf{S}=\frac{1}{2}\left[\begin{array}{cccc}
1 & 2 & 0 & 1 \\
1 & 0 & 0 & -1 \\
0 & 0 & 2 & 0 \\
1 & 0 & 0 & 1
\end{array}\right], S^{-1}=\left[\begin{array}{cccc}
0 & 1 & 0 & 1 \\
1 & 0 & 0 & -1 \\
0 & 0 & 1 & 0 \\
0 & -1 & 0 & 1
\end{array}\right]
$$

to obtain

$$
\mathrm{S}^{-1} \mathrm{AS}=\left[\begin{array}{cccc}
M+1 & 0 & 0 & 0 \\
0 & M & 0 & 0 \\
0 & 0 & M & 0 \\
0 & 0 & 0 & M-1
\end{array}\right], \mathrm{S}^{-1} \mathrm{BS}=\frac{1}{2}\left[\begin{array}{cccc}
0 & 0 & 2 & 0 \\
0 & 0 & 0 & 0 \\
1 & 0 & 0 & 1 \\
0 & 0 & 2 & 0
\end{array}\right]
$$

where $\mathrm{S}^{-1} \boldsymbol{q}=\boldsymbol{R}=[p+u, \rho-p, v, p-u]^{T}$ represents the characteristic variables. We recognize the convective entropy $\left(R_{2}\right)$ and vorticity waves $\left(R_{3}\right)$, respectively, and the co- $\left(R_{1}\right)$ and counter-propagating $\left(R_{4}\right)$ sounds waves through which the complete physical scenario can be understood.

However, the similarity transformation also shows that A and B can be transformed such as to become symmetric simultaneously by multiplication with a positive definite diagonal matrix. Within the context of the present work this has the consequence that Eqs.(1)-(2) forms a strongly well-posed hyperbolic system [20] implying that the well-posedness of Eqs.(1)-(2) is unaffected by the addition of low order terms. As we shall see shortly, lack of strong well-posedness can have serious consequences and make the construction of convergent numerical schemes impossible due to inherent instabilities of the system of equations.

3. An Analysis of the Split-Field PML Method. Following the line of thought initiated in [10] for the development of perfectly matched layers (PML) for electromagnetics, $\mathrm{Hu}$ [18] recently proposed a splitfield PML scheme for the two-dimensional linearized Euler equations as given in Eqs.(1)-(2). Different from the approach of [10], in which only some of the field components are split, in [18] all the field components of $q$ are split to arrive at a set of equations to be solved in the layer of the form

$$
\frac{\partial \boldsymbol{q}^{s}}{\partial t}+\mathrm{A}^{s} \frac{\partial \boldsymbol{q}^{s}}{\partial x}+\mathrm{B}^{s} \frac{\partial \boldsymbol{q}^{s}}{\partial y}+\mathrm{C}^{s} \boldsymbol{q}^{s}=0
$$

where $\boldsymbol{q}^{s}=\left[\rho_{1}, \rho_{2}, u_{1}, u_{2}, v_{1}, v_{2}, p_{1}, p_{2}\right]^{T}$, such that $\rho=\rho_{1}+\rho_{2}$ and likewise for the velocity components and the pressure. The $8 \times 8$ matrices in Eq. (3) become [18] 


$$
\mathrm{A}^{s}=\left[\begin{array}{cccccccc}
M & M & 1 & 1 & 0 & 0 & 0 & 0 \\
0 & 0 & 0 & 0 & 0 & 0 & 0 & 0 \\
0 & 0 & 0 & 0 & 0 & 0 & 1 & 1 \\
0 & 0 & M & M & 0 & 0 & 0 & 0 \\
0 & 0 & 0 & 0 & 0 & 0 & 0 & 0 \\
0 & 0 & 0 & 0 & M & M & 0 & 0 \\
0 & 0 & 1 & 1 & 0 & 0 & M & M \\
0 & 0 & 0 & 0 & 0 & 0 & 0 & 0
\end{array}\right], \mathrm{B}^{s}=\left[\begin{array}{llllllll}
0 & 0 & 0 & 0 & 0 & 0 & 0 & 0 \\
0 & 0 & 0 & 0 & 1 & 1 & 0 & 0 \\
0 & 0 & 0 & 0 & 0 & 0 & 0 & 0 \\
0 & 0 & 0 & 0 & 0 & 0 & 0 & 0 \\
0 & 0 & 0 & 0 & 0 & 0 & 1 & 1 \\
0 & 0 & 0 & 0 & 0 & 0 & 0 & 0 \\
0 & 0 & 0 & 0 & 0 & 0 & 0 & 0 \\
0 & 0 & 0 & 0 & 1 & 1 & 0 & 0
\end{array}\right],
$$

while $\mathrm{C}^{s}=\operatorname{diag}\left(\sigma_{x}, \sigma_{y}, \sigma_{x}, \sigma_{x}, \sigma_{y}, \sigma_{x}, \sigma_{x}, \sigma_{y}\right)$ represents the diagonal matrix responsible for the dissipation of the waves.

At first the use of split variables may seem perfectly legal since for $\sigma_{x}=\sigma_{y}=0$ the original equations are recovered by adding the equations for the split fields. This reasoning, however, is faulty as we shall show in the following.

Let us first address the issue of well-posedness of the split system of equations, Eqs. (3)-(4) and recall that the question of well-posedness of the system is unaffected by the low order term, $\mathrm{C}^{s} q^{s}$, which we therefore neglect in the subsequent analysis. We first consider the diagonalizing similarity transform of $A^{s}$ given as

$\mathrm{S}=\left[\begin{array}{cccccccc}0 & 0 & 0 & -1 & 1 & 0 & 1 & 1 \\ 0 & 0 & 0 & 1 & 0 & 0 & 0 & 0 \\ 0 & 0 & -1 & 0 & \frac{1}{M-1} & 0 & 0 & \frac{1}{M+1} \\ 0 & 0 & 1 & 0 & \frac{-M}{M-1} & 0 & 0 & \frac{M}{M+1} \\ 0 & -1 & 0 & 0 & 0 & 0 & 0 & 0 \\ 0 & 1 & 0 & 0 & 0 & 1 & 0 & 0 \\ -1 & 0 & 0 & 0 & 1 & 0 & 0 & 1 \\ 1 & 0 & 0 & 0 & 0 & 0 & 0 & 0\end{array}\right], S^{-1}=\left[\begin{array}{cccccccc}0 & 0 & 0 & 0 & 0 & 0 & 0 & 1 \\ 0 & 0 & 0 & 0 & 1 & 0 & 0 & 0 \\ 0 & 0 & \frac{-M^{2}}{M^{2}-1} & \frac{-1}{M^{2}-1} & 0 & 0 & \frac{M}{M^{2}-1} & \frac{M}{M^{2}-1} \\ 0 & 1 & 0 & 0 & 0 & 0 & 0 & 0 \\ 0 & 0 & -\frac{1}{2} & -\frac{1}{2} & 0 & 0 & \frac{1}{2} & \frac{1}{2} \\ 0 & 0 & 0 & 0 & 1 & 1 & 0 & 0 \\ 1 & 1 & 0 & 0 & 0 & 0 & -1 & -1 \\ 0 & 0 & \frac{1}{2} & \frac{1}{2} & 0 & 0 & \frac{1}{2} & \frac{1}{2}\end{array}\right]$.

to obtain $\mathrm{S}^{-1} \mathrm{~A}^{s} \mathrm{~S}=\operatorname{diag}(0,0,0,0, M-1, M, M, M+1)$, i.e., 4 zero eigenvalues have been introduced as a consequence of the splitting. Now, if $\mathrm{S}$ and $\mathrm{S}^{-1}$ cannot transform $\mathrm{B}^{s}$ into a matrix that can be made symmetric by multiplication with a positive definite diagonal matrix, then $\mathrm{A}^{s}$ and $\mathrm{B}^{s}$ cannot be symmetrized simultaneously [21]. Indeed, we obtain

$$
\mathrm{S}^{-1} \mathrm{~B}^{s} \mathrm{~S}=\left[\begin{array}{cccccccc}
0 & 0 & 0 & 0 & 0 & 1 & 0 & 0 \\
0 & 0 & 0 & 0 & -1 & 0 & 0 & -1 \\
0 & 0 & 0 & 0 & 0 & \frac{M}{M^{2}-1} & 0 & 0 \\
0 & 0 & 0 & 0 & 0 & 1 & 0 & 0 \\
0 & 0 & 0 & 0 & 0 & \frac{1}{2} & 0 & 0 \\
0 & 0 & 0 & 0 & 1 & 0 & 0 & 1 \\
0 & 0 & 0 & 0 & 0 & 0 & 0 & 0 \\
0 & 0 & 0 & 0 & 0 & \frac{1}{2} & 0 & 0
\end{array}\right]
$$

which, due to the zeros in the left half, clearly can not be made symmetric. This observation, however, is not conclusive in terms of lack of strong well-posedness of Eqs.(3)-(4), but it remains a bad sign, since the split set of equations has lost an important symmetry property as compared to the original un-split linearized Euler equations, Eqs.(1)-(2). 
To continue the analysis we shall focus the attention on the Cauchy problem, i.e., neglect the effect of the boundary conditions in Eqs.(3)-(4). We introduce the spatial Fourier transform of $\boldsymbol{q}^{\boldsymbol{s}}$ as

$$
\boldsymbol{q}^{s}(x, y, t)=\int_{-\infty}^{\infty} \int_{-\infty}^{\infty} \hat{\boldsymbol{q}}^{s}\left(k_{x}, k_{y}, t\right) e^{i\left(k_{x} x+k_{y} y\right)} d k_{x} d k_{y}
$$

where $\hat{\boldsymbol{q}}^{s}=\left[\hat{\rho}_{1}, \hat{\rho}_{2}, \hat{u}_{1}, \hat{u}_{2}, \hat{v}_{1}, \hat{v}_{2}, \hat{p}_{1}, \hat{p}_{2}\right]^{T}$ represents the Fourier coefficients of the split field components to arrive at the initial value problem

$$
\frac{\partial \hat{\boldsymbol{q}}^{s}}{\partial t}=\mathrm{P}\left(k_{x}, k_{y}\right) \hat{\boldsymbol{q}}^{s}
$$

where the symbol, $\mathrm{P}\left(k_{x}, k_{y}\right)$, is given as

$$
\mathrm{P}\left(k_{x}, k_{y}\right)=-i\left[\begin{array}{cccccccc}
M k_{x} & M k_{x} & k_{x} & k_{x} & 0 & 0 & 0 & 0 \\
0 & 0 & 0 & 0 & k_{y} & k_{y} & 0 & 0 \\
0 & 0 & 0 & 0 & 0 & 0 & k_{x} & k_{x} \\
0 & 0 & M k_{x} & M k_{x} & 0 & 0 & 0 & 0 \\
0 & 0 & 0 & 0 & 0 & 0 & k_{y} & k_{y} \\
0 & 0 & 0 & 0 & M k_{x} & M k_{x} & 0 & 0 \\
0 & 0 & k_{x} & k_{x} & 0 & 0 & M k_{x} & M k_{x} \\
0 & 0 & 0 & 0 & k_{y} & k_{y} & 0 & 0
\end{array}\right] .
$$

Integration of Eqs.(5)-(6) is achieved by first realizing that the evolution of the individual split components depends only on the un-split variables. Hence, by obtaining the solution of the Cauchy problem of Eqs.(1)-(2), given on the form

$$
\begin{aligned}
& \frac{\partial \hat{\rho}}{\partial t}=-i M k_{x} \hat{\rho}-i k_{x} \hat{u}-i k_{y} \hat{v} \\
& \frac{\partial \hat{u}}{\partial t}=-i M k_{x} \hat{u}-i k_{x} \hat{p} \\
& \frac{\partial \hat{v}}{\partial t}=-i M k_{x} \hat{v}-i k_{y} \hat{p} \\
& \frac{\partial \hat{p}}{\partial t}=-i M k_{x} \hat{p}-i k_{x} \hat{u}-i k_{y} \hat{v}
\end{aligned}
$$

we may obtain the solution for the split field variables by introducing the solutions of the un-split variables into Eqs.(5)-(6).

Considering the initial conditions

$$
\hat{\boldsymbol{q}}(0)=\left[\hat{\rho}_{0}, \hat{u}_{0}, \hat{v}_{0}, \hat{p}_{0}\right]^{T}
$$

we obtain the solution to Eq.(7) as

$$
\hat{\boldsymbol{q}}(t)=\boldsymbol{a} e^{-i\left(M k_{x}-\nu\right) t}+\boldsymbol{b} e^{-i\left(M k_{x}+\nu\right) t}+\boldsymbol{c} e^{-i M k_{x} t}
$$

with the three vectors $a=\left[a_{\rho}, a_{u}, a_{v}, a_{p}\right]^{T}, \boldsymbol{b}=\left[b_{\rho}, b_{u}, b_{v}, b_{p}\right]^{T}$ and $c=\left[c_{\rho}, c_{u}, c_{v}, c_{p}\right]^{T}$, having the entries 


$$
\boldsymbol{a}=\frac{\mu-\hat{p}_{0} \nu}{2 \nu^{2}}\left[\begin{array}{c}
-\nu \\
k_{x} \\
k_{y} \\
-\nu
\end{array}\right], \boldsymbol{b}=\frac{\mu+\hat{p}_{0} \nu}{2 \nu^{2}}\left[\begin{array}{c}
\nu \\
k_{x} \\
k_{y} \\
\nu
\end{array}\right], \boldsymbol{c}=\frac{1}{\nu^{2}}\left[\begin{array}{c}
0 \\
\hat{u}_{0} \nu^{2}-k_{x} \mu \\
\hat{v}_{0} \nu^{2}-k_{y} \mu \\
0
\end{array}\right] .
$$

Here we have

$$
\nu=\sqrt{k_{x}^{2}+k_{y}^{2}}, \mu=\hat{u}_{0} k_{x}+\hat{v}_{0} k_{y},
$$

and we have utilized that $\hat{\rho}_{0}=\hat{p}_{0}$ for consistency. In Eq.(8) we immediately recognize the three types of waves, inherent in the linearized Euler equations, giving rise to three different wave speeds. Moreover, we note that $\boldsymbol{a}$ and $\boldsymbol{b}$ as well as $\boldsymbol{c}$ are bounded for all values of $k_{x}$ and $k_{y}$ confirming that Eqs.(1)-(2) constitute a strongly well-posed problem for which the solution can be bounded up to exponential growth in time by the norm of the initial data.

Let us now continue in order to arrive at the solution of the Cauchy problem for $\hat{\boldsymbol{q}}_{2}^{s}=\left[\hat{\rho}_{2}, \hat{u}_{2}, \hat{v}_{2}, \hat{p}_{2}\right]^{T}$, bearing in mind that we could equally well have chosen the other set of equations. The dynamics of these 4 variables are described by the system

$$
\begin{aligned}
& \frac{\partial \hat{\rho}_{2}}{\partial t}=-i k_{y} \hat{v}, \\
& \frac{\partial \hat{u}_{2}}{\partial t}=-i M k_{x} \hat{u}, \\
& \frac{\partial \hat{v}_{2}}{\partial t}=-i M k_{x} \hat{v}, \\
& \frac{\partial \hat{p}_{2}}{\partial t}=-i k_{y} \hat{v} .
\end{aligned}
$$

Integrating the solution, Eq.(8), we recover the solution to Eq.(9) on the form

$$
\hat{\boldsymbol{q}}_{2}(t)-\hat{\boldsymbol{q}}_{2}(0)=\boldsymbol{a}_{2}^{s} e^{-i \frac{M k_{\bar{I}}-\nu}{2} t}+\boldsymbol{b}_{2}^{s} e^{-i \frac{M k_{\bar{x}}+\nu}{2} t}+c_{2}^{s} e^{-i \frac{M k_{\bar{x}}}{2} t}
$$

where

$$
\boldsymbol{a}_{2}^{s}=-i \frac{\sin \left[\frac{M k_{x}-\nu}{2} t\right]}{\frac{M k_{x}-\nu}{2}}\left[\begin{array}{c}
k_{y} a_{v} \\
M k_{x} a_{u} \\
M k_{x} a_{v} \\
k_{y} a_{v}
\end{array}\right], \boldsymbol{b}_{2}^{s}=-i \frac{\sin \left[\frac{M k_{x}+\nu}{2} t\right]}{\frac{M k_{x}+\nu}{2}}\left[\begin{array}{c}
k_{y} b_{v} \\
M k_{x} b_{u} \\
M k_{x} b_{v} \\
k_{y} b_{v}
\end{array}\right],
$$

and

$$
c_{2}^{s}=-i \frac{\sin \left[\frac{M k_{x}}{2} t\right]}{\frac{M k_{x}}{2}}\left[\begin{array}{c}
k_{y} c_{v} \\
M k_{x} c_{u} \\
M k_{x} c_{v} \\
k_{y} c_{v}
\end{array}\right]
$$

For the split set of equations to be strongly well-posed we must ensure that the solution, Eq.(10), is bounded by the norm of the initial data for any choice of $k_{x}$ and $k_{y}$, or, in other words, $\boldsymbol{a}_{2}^{s}, \boldsymbol{b}_{2}^{s}$ and $\boldsymbol{c}_{2}^{s}$ must remain 
bounded for any combination of $k_{x}$ and $k_{y}$. It is easily verified that $\boldsymbol{a}_{2}^{s}$ and $\boldsymbol{b}_{2}^{s}$ indeed remain bounded for all values of $k_{x}$ and $k_{y}$ provided the mean flow is purely subsonic, i.e. $|M|<1$. However, absorbing boundary conditions are not necessary in the case of supersonic flow as reflections from the open boundary are unable to enter the computational domain and therefore will not cause any problems.

The situation for $c_{2}^{s}$ is very different. In the limit where $\left|M k_{x}\right| \rightarrow 0$ and $\left|k_{y}\right| \gg\left|M k_{x}\right|$ we can only bound two of the terms in $c_{2}^{s}$ as

$$
\left|k_{y} c_{v} \frac{\sin \left[\frac{M k_{x}}{2} t\right]}{\frac{M k_{x}}{2}}\right| \leq\left|c_{v} k_{y}\right| t,
$$

i.e, we recover terms that grow in time with a coefficient, $k_{y}$, which is unbounded. Hence, $\left\|\hat{\rho}_{2}\right\|$ and $\left\|\hat{p}_{2}\right\|$ cannot be bounded by the norm of the initial conditions, but rather depends also on the norm of the derivatives of the initial conditions, $\hat{u}_{0}$ and $\hat{v}_{0}$. Consequently, the split set of equations, Eq.(3), is only weakly well-posed with the solution depending not only on the initial conditions, but also on the smoothness of these data.

It is noteworthy that, as is the case for the split field perfectly matched layer methods of electromagnetics $[10,11]$, in the case where $\left|k_{y}\right|=0$ strong well-posedness is recovered. Hence, the one-dimensional version of the split field method for the perfectly matched layers of acoustics is valid and well suited for numerical solution.

The loss of derivatives is as such not a severe problem. However, contrary to strongly well-posed hyperbolic problems, it is well known that weakly well-posed systems may become ill-posed under low order perturbations [20], thus rendering the systems of equations inherently unstable and proper numerical solution impossible.

To see this, we introduce a low order perturbation of the form

$$
\mathrm{E} \hat{\boldsymbol{q}}^{s}=\left[\begin{array}{cccccccc}
0 & 0 & 0 & 0 & 0 & 0 & 0 & 0 \\
0 & 0 & 0 & 0 & \varepsilon & -\varepsilon & 0 & 0 \\
0 & 0 & 0 & 0 & 0 & 0 & 0 & 0 \\
0 & 0 & 0 & 0 & 0 & 0 & 0 & 0 \\
0 & 0 & 0 & 0 & 0 & 0 & 0 & 0 \\
0 & 0 & 0 & 0 & \varepsilon & -\varepsilon & 0 & 0 \\
0 & 0 & 0 & 0 & 0 & 0 & 0 & 0 \\
0 & 0 & 0 & 0 & \varepsilon & -\varepsilon & 0 & 0
\end{array}\right] \hat{\boldsymbol{q}}^{s}
$$

i.e. the perturbation corresponds to a small perturbation in the split field velocity component, $\hat{v}_{1}$ and $\hat{v}_{2}$, however maintaining that $\hat{v}=\hat{v}_{1}+\hat{v}_{2}$.

We consider the perturbed Cauchy problem

$$
\frac{\partial \hat{\boldsymbol{q}}^{s}}{\partial t}=\left(\mathrm{P}\left(k_{x}, k_{y}\right)+\mathrm{E}\right) \hat{\boldsymbol{q}}^{s}=\tilde{\mathrm{P}} \hat{\boldsymbol{q}}^{s}
$$

A necessary condition for the perturbed problem to remain well-posed is that the real parts of the eigenvalues of $\tilde{\mathrm{P}}$ remain in the left half plane for any choice of $k_{x}$ and $k_{y}$ and, preferably, also for any choice of $\varepsilon$ and $M$. The 4 eigenvalues of $\tilde{\mathrm{P}}$ are given as

$$
\lambda_{1}=\lambda_{2}=\lambda_{3}=0, \lambda_{4}=-i k_{x} M
$$


while the remaining 4 eigenvalues appear as the roots of a complex polynomial

$$
P_{4}(\lambda)=\alpha_{4} \lambda^{4}+\alpha_{3} \lambda^{3}+\alpha_{2} \lambda^{2}+\alpha_{1} \lambda+\alpha_{0}=0
$$

in which the coefficients are given as

$$
\begin{gathered}
\alpha_{4}=1, \alpha_{3}=\varepsilon+i 3 M k_{x}, \alpha_{2}=k_{x}^{2}+k_{y}^{2}-3 M^{2} k_{x}^{2}+i \varepsilon\left(k_{y}+2 k_{x} M\right) \\
\alpha_{1}=\varepsilon\left(k_{x}^{2}+2 k_{y}^{2}-3 M k_{x} k_{y}-M^{2} k_{x}^{2}\right)+i M k_{x}\left(k_{x}^{2}-M^{2} k_{x}^{2}+k_{y}^{2}\right) \\
\alpha_{0}=i 2 \varepsilon M k_{x} k_{y}^{2}(1-M) .
\end{gathered}
$$

Rather than solving the complex polynomial to obtain the expressions for the eigenvalues, we shall recall the Routh-Hurwitz criteria expressing that all the roots, $\lambda$, of an $n$ 'th-order polynomial, $P_{n}(\lambda)$, lies in the left half plane if and only if the roots of the $(n-1)$ 'th-order polynomial

$$
P_{n-1}(\lambda)=\left[\alpha_{n} \alpha_{n-1}^{*}+\alpha_{n-1} \alpha_{n}^{*}-\alpha_{n} \alpha_{n}^{*} \lambda\right] P_{n}(\lambda)+\alpha_{n}^{2} \lambda \sum_{i=0}^{n}(-1)^{n-i} \alpha_{i}^{*} \lambda^{i}
$$

lies in the left half plane and the real part of $\alpha_{n-1} / \alpha_{n}$ is positive. Hence, by successively applying this result we arrive at criteria under which the perturbed initial value problem remains well-posed.

The first condition immediately yields the requirement that $\operatorname{Re}\left(\alpha_{3} / \alpha_{4}\right)=\varepsilon>0$ which certainly is a bad sign since we can not in general control the sign of the perturbation. However, one additional application of the Routh-Hurwitz criteria results in a condition for well-posedness as

$$
\left|M k_{x}\right|>\left|k_{y}\right|
$$

This condition is very similar to the limit for boundedness of $c_{2}^{s}$, Eq.(11), and confirms that the weakly well-posed system, arrived at by splitting the linearized Euler equations to facilitate the development of the perfectly matched layers, becomes ill-posed under low order perturbations. We should note that there is nothing particular about the low order perturbation, E. Indeed, ill-posedness can be shown, using the technique outlined above, for perturbations of the velocity components as well as of the density and pressure components.

Due to finite precision, low order perturbations will always exist in actual implementations of the split field equations, i.e., problems with maintaining stability of these schemes should be expected. Indeed, this is exactly what was reported in $[18,19]$ where it was found that applying a filter in the PML layers was necessary to maintain stability. An indication of why the filter has a stabilizing effect for this problem is provided by the condition for boundedness, Eq.(11). Indeed, if the filter is sufficiently strong as to ensure that $\left|M k_{x}\right|>\left|k_{y}\right|$ for all values of $\left|k_{x}\right|$ and $\left|k_{y}\right|$, e.g. by enforcing a strong filter along $y$, the system remains well-posed and, as a consequence, the scheme might recover stability or at least postpone the effects of the instability.

4. The Construction of Well-posed PML Methods. The inherent instability appearing as a result of the splitting of the linearized Euler equations illustrates the care that has to be exercised in devising absorbing layers for such types of equations. 
The weakly well-posedness and associated ill-posedness under small perturbations of the split-field PML equations was recently shown [11] also for the original PML method as proposed in [10] and several successful attempts have been made to formulate strongly well-posed PML methods for the equations of electromagnetics $[12,13,14,16,17]$. Hence, rather than attempting an ab initio development of perfectly absorbing layers for the linearized Euler equations, we shall utilize this recent development to arrive at the sought after well behaved methods.

A strongly well-posed PML method for Maxwell's equations is proposed in [17] and tested numerically in [16] and we shall base the remaining part of this paper on this particular formulation. We should emphasize though that alternative well-posed formulations might equally well be employed as the basis of the development of the PML methods for the equations of acoustics.

In what remains we restrict the attention to the case of a purely subsonic free stream, i.e. $|M|<1$ in Eq.(1). This poses no restrictions on the analysis and the applicability of the proposed schemes as the situation for $|M|>1$ is trivial since all information is leaving the computational domain and, as information is prohibited from propagating upstream, reflections cannot enter the domain and interfere with the computed results.

4.1. The Quiescent Case. Let us first consider the simple case of a quiescent free-stream, i.e. $M=0$, for which the equations are given as

$$
\begin{aligned}
& \frac{\partial \rho}{\partial t}=-\frac{\partial u}{\partial x}-\frac{\partial v}{\partial y} \\
& \frac{\partial u}{\partial t}=-\frac{\partial p}{\partial x} \\
& \frac{\partial v}{\partial t}=-\frac{\partial p}{\partial y}, \\
& \frac{\partial p}{\partial t}=-\frac{\partial u}{\partial x}-\frac{\partial v}{\partial y}
\end{aligned}
$$

appearing directly from Eqs.(1)-(2).

We propose to consider an absorbing layer for the quiescent linearized Euler equations of the form

$$
\begin{aligned}
& \frac{\partial \rho}{\partial t}=-\frac{\partial u}{\partial x}-\frac{\partial v}{\partial y}-\varepsilon^{\prime} Q_{x}-\mu^{\prime} Q_{y}, \\
& \frac{\partial u}{\partial t}=-\frac{\partial p}{\partial x}-2 \varepsilon u-\varepsilon P_{x}, \\
& \frac{\partial v}{\partial t}=-\frac{\partial p}{\partial y}-2 \mu v-\mu P_{y}, \\
& \frac{\partial p}{\partial t}=-\frac{\partial u}{\partial x}-\frac{\partial v}{\partial y}-\varepsilon^{\prime} Q_{x}-\mu^{\prime} Q_{y}, \\
& \frac{\partial P_{x}}{\partial t}=\varepsilon u, \frac{\partial Q_{x}}{\partial t}=-\varepsilon Q_{x}+u, \\
& \frac{\partial P_{y}}{\partial t}=\mu v, \frac{\partial Q_{y}}{\partial t}=-\mu Q_{y}+v .
\end{aligned}
$$

Here, $\varepsilon=\varepsilon(x)$ and $\mu=\mu(y)$ signifies the non-dimensional damping parameters. We immediately note that since the Euler equations are altered by the introduction of low-order terms only, the system of partial differential equations is well-posed by construction while the additional freedom required for obtaining the 
sought after properties of the matched layers are introduced through 4 additional equations describing the development of the artificial fields, $P_{x}$ and $Q_{x}$, along $x$ and, likewise, $P_{y}$ and $Q_{y}$, along $y$. In general, we assume that the absorbing region is outside a square bounded by $|x|=a$ and $|y|=b$ while the specification of the parameters, $\varepsilon$ and $\mu$ remains open at this point in time.

To come to an understanding of the properties of this absorbing layer, we follow the analysis introduced in [11] and study the behavior of a plane wave hitting the layer interface, which we assume is positioned at $x=0$. As the system we are dealing with is purely linear, it poses no restrictions to consider only the behavior of plane waves, since any type of excitation can be decomposed into a superposition of such plane waves of the form

$$
\left[\begin{array}{l}
\rho \\
u \\
v \\
p
\end{array}\right]=\left[\begin{array}{c}
1 \\
\alpha \\
\beta \\
1
\end{array}\right] e^{i \omega(t-\alpha x-\beta y)}
$$

where $\alpha^{2}+\beta^{2}=1$ represents the arbitrary angle of incidence and $\omega$ signifies the normalized frequency of the incoming wave.

We shall seek solutions inside a layer in the $x$-direction, i.e $\mu(y)=0$ in Eq.(13), of the form

$$
\left[\begin{array}{c}
\rho \\
u \\
v \\
p \\
Q_{x} \\
P_{x}
\end{array}\right]=\left[\begin{array}{c}
\tilde{\rho}(x) \\
\tilde{u}(x) \\
\tilde{v}(x) \\
\tilde{p}(x) \\
\tilde{Q}_{x}(x) \\
\tilde{P}_{x}(x)
\end{array}\right] e^{i \omega(t-\beta y)}
$$

and shall obtain the full solutions for the fields inside the layer.

Introducing Eq.(15) into Eq.(13) yields the equations

$$
\tilde{\rho}=\tilde{p}, \tilde{v}=\beta \tilde{p}, \tilde{Q}_{x}=\frac{1}{\psi} \tilde{u}, \tilde{P}_{x}=\frac{\varepsilon}{i \omega} \tilde{u},
$$

thus expressing 4 of the 6 variables in terms of $\tilde{u}$ and $\tilde{p}$. The latter are governed by the coupled equations

$$
\frac{d}{d x} \tilde{p}=-\frac{\psi^{2}}{i \omega} \tilde{u}, \frac{d}{d x}[\psi \tilde{u}]=-i \omega \psi \alpha^{2} \tilde{p}
$$

where we have introduced $\psi(x)=i \omega+\varepsilon(x)$. Combining these two equations yields a second order variable coefficient ODE for $\tilde{p}$ on the form

$$
\frac{d}{d x}\left(\frac{1}{\psi} \frac{d}{d x} \tilde{p}\right)=\psi \alpha^{2} \tilde{p}
$$

which has an analytical solution given by

$$
\tilde{p}(x)=A e^{\alpha \int_{0}^{x} \psi(\eta) d \eta}+B e^{-\alpha \int_{0}^{x} \psi(\eta) d \eta}
$$

through which we immediately obtain the solution to $\tilde{u}$ as 


$$
\tilde{u}(x)=-\frac{i \omega}{\psi^{2}} \frac{d}{d x} \tilde{p}(x)=-\alpha \frac{i \omega}{\psi}\left(A e^{\alpha \int_{0}^{x} \psi(\eta) d \eta}-B e^{-\alpha \int_{0}^{x} \psi(\eta) d \eta}\right)
$$

The remaining fields are then given from this using Eq.(16).

The specification $A$ and $B$ naturally depends on the boundary conditions we choose to impose and, indeed, there are several ways of doing so. We shall assume that the layer has a finite width, $d$, and shall hence need to impose boundary conditions at $x=0$ and $x=d$. For the solution of hyperbolic systems it is most natural to impose characteristic boundary conditions by specifying the incoming characteristics. This amounts to requiring that $R_{1}=p+u$ remains continuous across the interface $x=0$ while $R_{4}=p-u=0$ at $x=d$, i.e. no information is entering the layer. Imposing these boundary conditions, using Eq.(14), implies

$$
\tilde{p}(0)+\tilde{u}(0)=1+\alpha, \tilde{p}(d)-\tilde{u}(d)=0,
$$

from which we arrive at $(\alpha \neq 0)$

$$
A=\frac{\alpha-\gamma}{\alpha+\gamma} e^{-2 \alpha I} B, B=\frac{1}{1+\frac{\alpha-\gamma}{\alpha+\gamma} \frac{1-\alpha}{1+\alpha} e^{-2 \alpha I}},
$$

where

$$
\gamma=\frac{\varepsilon(d)+i \omega}{i \omega}, I=\int_{0}^{d} \psi(\eta) d \eta=i \omega d+\int_{0}^{d} \varepsilon(\eta) d \eta .
$$

Combining Eq.(16) with Eqs.(17)-(19) yields the complete solution inside the layer,

$$
\begin{aligned}
\rho(x, y, t) & =B\left[1+\frac{\alpha-\gamma}{\alpha+\gamma} e^{2 i \omega \alpha(x-d)} e^{-2 \alpha \int_{x}^{d} \varepsilon(\eta) d \eta}\right] e^{i \omega(t-\alpha x-\beta y)} e^{-\alpha \int_{0}^{x} \varepsilon(\eta) d \eta} \\
u(x, y, t) & =-B \alpha \frac{i \omega}{\varepsilon(x)+i \omega}\left[1-\frac{\alpha-\gamma}{\alpha+\gamma} e^{2 i \omega \alpha(x-d)} e^{-2 \alpha \int_{x}^{d} \varepsilon(\eta) d \eta}\right] e^{i \omega(t-\alpha x-\beta y)} e^{-\alpha \int_{0}^{x} \varepsilon(\eta) d \eta} \\
v(x, y, t) & =\beta B\left[1+\frac{\alpha-\gamma}{\alpha+\gamma} e^{2 i \omega \alpha(x-d)} e^{-2 \alpha \int_{x}^{d} \varepsilon(\eta) d \eta}\right] e^{i \omega(t-\alpha x-\beta y)} e^{-\alpha \int_{0}^{x} \varepsilon(\eta) d \eta} \\
p(x, y, t) & =B\left[1+\frac{\alpha-\gamma}{\alpha+\gamma} e^{2 i \omega \alpha(x-d)} e^{-2 \alpha \int_{x}^{d} \varepsilon(\eta) d \eta}\right] e^{i \omega(t-\alpha x-\beta y)} e^{-\alpha \int_{0}^{x} \varepsilon(\eta) d \eta} \\
P_{x}(x, y, t) & =-B \alpha \frac{\varepsilon}{\varepsilon(x)+i \omega}\left[1-\frac{\alpha-\gamma}{\alpha+\gamma} e^{2 i \omega \alpha(x-d)} e^{-2 \alpha \int_{x}^{d} \varepsilon(\eta) d \eta}\right] e^{i \omega(t-\alpha x-\beta y)} e^{-\alpha \int_{0}^{x} \varepsilon(\eta) d \eta} \\
Q_{x}(x, y, t) & =B \alpha \frac{i \omega}{(\varepsilon(x)+i \omega)^{2}}\left[1-\frac{\alpha-\gamma}{\alpha+\gamma} e^{2 i \omega \alpha(x-d)} e^{-2 \alpha \int_{x}^{d} \varepsilon(\eta) d \eta}\right] e^{i \omega(t-\alpha x-\beta y)} e^{-\alpha \int_{0}^{x} \varepsilon(\eta) d \eta}
\end{aligned}
$$

Since $\left|\frac{i \omega}{\epsilon+i \omega}\right|<1$ all components in the layer are bounded by $|p|$ which is bounded like $\alpha \frac{\partial|p|}{\partial x} \leq 0$ with equality only for gazing waves, i.e. $\alpha=0$. Thus, all waves are damped independent of frequency and angle of incidence as should be required by a truly perfectly matched layer.

We observe that the fields at the layer interface, $x=0$, in general are discontinuous with a jump proportional

$$
\frac{1 \pm \frac{\alpha-\gamma}{\alpha+\gamma} e^{-2 i \omega \alpha d} e^{-2 \alpha I}}{1+\frac{\alpha-\gamma}{\alpha+\gamma} \frac{1-\alpha}{1+\alpha} e^{-2 \alpha I}}
$$

which, however, is exponentially small.

Naturally, an analysis equivalent to the above can be completed for a PML layer in the $y$-direction while a corner region, in which $\varepsilon>0$ as well as $\mu>0$, can be analyzed using separation of variables, yielding results similar to those obtained in the layer above. 
4.1.1. A Numerical Example. In order to confirm the theoretical analysis put forward in the previous section and study the efficiency of this new PML method, we have implemented the scheme on an equidistant grid using a 4'th order, centered, finite-difference scheme with 3'rd order closure for stability in space, while we use a 4 'th order Runge-Kutta scheme for advancing the system, Eq.(12), in time. The time step, $\Delta t$, is chosen to be well below the stability limit.

We note that contrary to the scheme proposed in [18], there is no need for applying a filter to maintain stability and, to emphasize this point, we have not used any filters in the present work.

The initial conditions are taken from a benchmark problem of computational aeroacoustics found in [22], namely

$$
\begin{aligned}
& \rho(x, y)=e^{-(\ln 2) \frac{\left(x-x_{a}\right)^{2}+\left(y-v_{a}\right)^{2}}{\delta_{a}^{2}}}+0.1 e^{-(\ln 2) \frac{\left(x-x_{b}\right)^{2}+\left(y-y_{b}\right)^{2}}{\delta_{b}^{2}}}, \\
& u(x, y)=0.05\left(y-y_{b}\right) e^{-(\ln 2) \frac{\left(x-x_{b}\right)^{2}+\left(y-y_{b}\right)^{2}}{\delta_{b}^{2}}}, \\
& v(x, y)=-0.05\left(x-x_{b}\right) e^{-(\ln 2) \frac{\left(x-x_{b}\right)^{2}+\left(y-\nu_{b}\right)^{2}}{\delta_{b}^{2}}}, \\
& p(x, y)=e^{-(\ln 2) \frac{\left(x-x_{a}\right)^{2}+\left(y-y_{a}\right)^{2}}{\delta_{a}^{2}}}
\end{aligned}
$$

where $\left(x_{a}, y_{a}\right)$ signifies the center of the initial sound pulse of width $\delta_{a}$, while $\left(x_{b}, y_{b}\right)$ refers to the center of the initial vorticity and entropy pulse of width $\delta_{b}$. The analytic solution to this problem may be obtained by exploiting the axis-symmetry of the initial conditions together with the use of Fourier transformation. The exact expressions for the solution are given in [22].

The profiles, $\varepsilon(x)$ and $\mu(y)$, required in the specification of the scheme, Eq.(13), are taken to be of the form

$$
\varepsilon(x)=C_{x}\left(\frac{|x-a|}{x_{P M L}}\right)^{n}, \mu(y)=C_{y}\left(\frac{|y-b|}{y_{P M L}}\right)^{n} .
$$

Here we have assumed that the computational domain in which Eq.(12) is solved is bounded by $|x| \leq a$ and $|y| \leq b$ while $x_{P M L}$ and $y_{P M L}$ refers to the width of the absorbing layers along $x$ and $y$, respectively. The constants, $C_{x}, C_{y}$ and $n$, controls the strength of the layer and we have chosen these parameters as $C_{x}=C_{y}=2$ and $n=3$. The auxiliary equations of Eq.(13) are advanced in time using the same scheme and time-step as for Eq.(12).

We consider the problem in the computational domain $(x, y) \in[-50,50]^{2}$ with the absorbing layers outside and position the acoustics pulse at $\left(x_{a}, y_{a}\right)=(-25,0)$ with a width of $\delta_{a}=3$ while the nonpropagating vorticity/entropy pulse is positioned at $\left(x_{b}, y_{b}\right)=(25,0)$ with a width of $\delta_{b}=4$. The absorbing layers are terminated using characteristic boundary conditions as discussed during the analysis of the scheme.

In Fig. 1 we show the pressure field at various times as computed using $\Delta x=\Delta y=1$ and $\Delta t=1$ and $x_{P M L}=y_{P M L}=10$, i.e. 10 computational cells in the absorbing layer.

As expected from the analysis, the sound wave propagates undisturbed out of the computational domain with no visible reflections. The high frequency noise visible on the contours is a consequence of the accuracy of the scheme and the lack of filtering, rather than a result of reflections as can also be observed on Fig. 2, where we show the $u$-velocity field propagating undisturbed out of the computational domain.

To verify the dependency of the efficiency of the absorbing layer on the width of the layer, we have computed the maximum pressure error along the line $x=-48$ as a function of time. In Fig. 3 we show 
a)

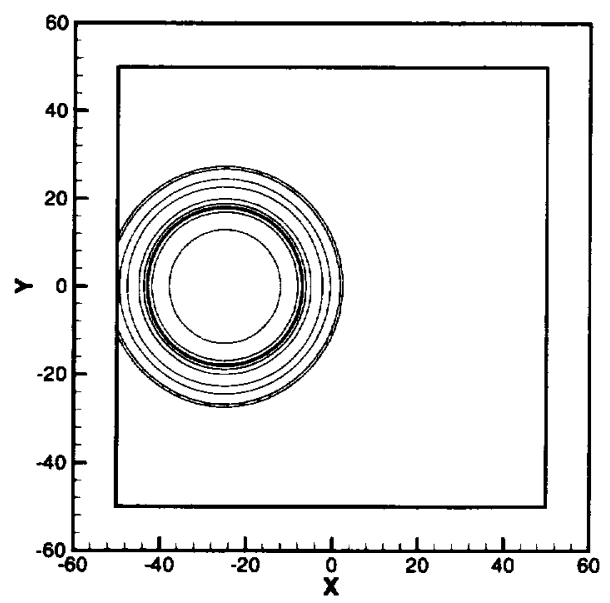

c)

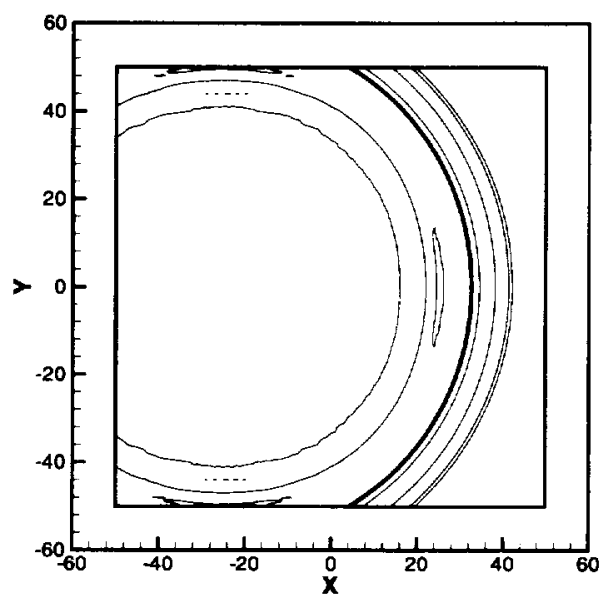

b)

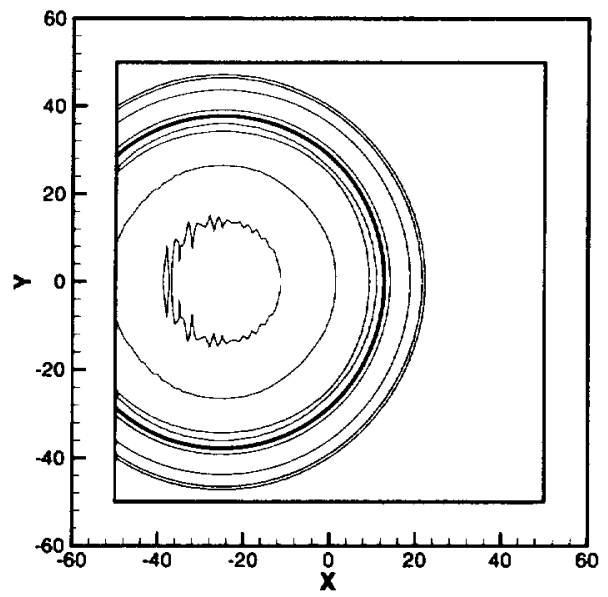

d)

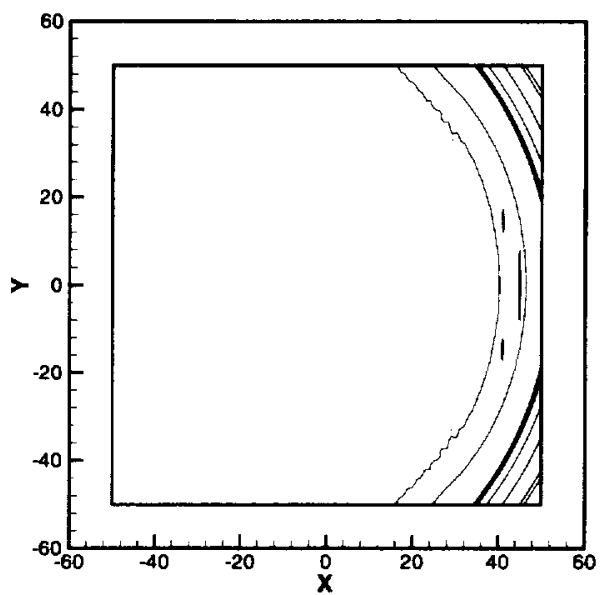

FIG. 1. Pressure contours for a sound pulse, propagating in a quiescent medium. The contour levels are $\pm 0.1, \pm 0.05, \pm 0.01$ and \pm 0.005 with the computed result given at $t=20$ (a), $t=40$ (b), $t=60$ (c) and $t=80$ (d).

the development of the pressure error for various layer widths as compared with using only characteristic boundary conditions to terminate the computational domain.

Indeed, as expected, we see that even for a layer of only 6 cells the PML scheme out-perform the characteristic $\mathrm{BC}$ in terms of accuracy while increasing the width of the layer yields a significant increase in accuracy.

As compared to the scheme put forward in [18] we observe a slight increase in the maximum error which is consistent with the observations made in [16] comparing the split and un-split PML methods for Maxwell's equations. A direct comparison, however, is difficult due to differences in the computational scheme and boundary conditions. We emphasize, though, that the present results are arrived at without the use of filtering, thus confirming the stability of the scheme given in Eq.(13) and the associated analysis of well-posedness and decaying properties of the fields inside the layers.

4.2. The Convecting Case. While the development of PML methods for the quiescent equations of acoustics relies on the analogy with the equations of electromagnetics, no such connection is possible in the 
a)

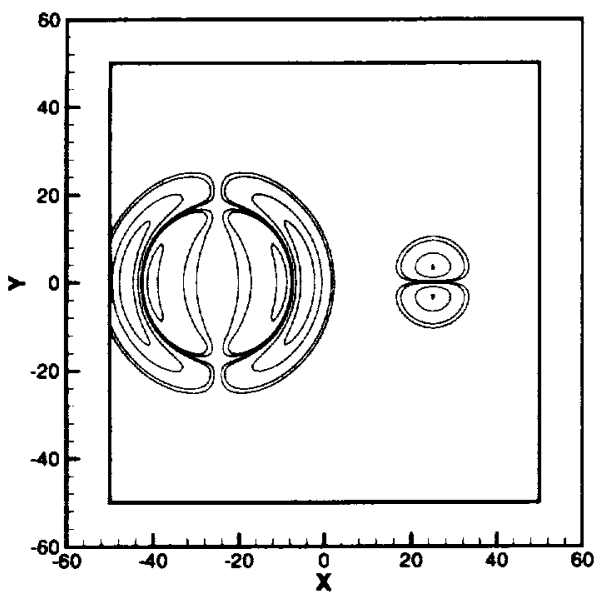

c)

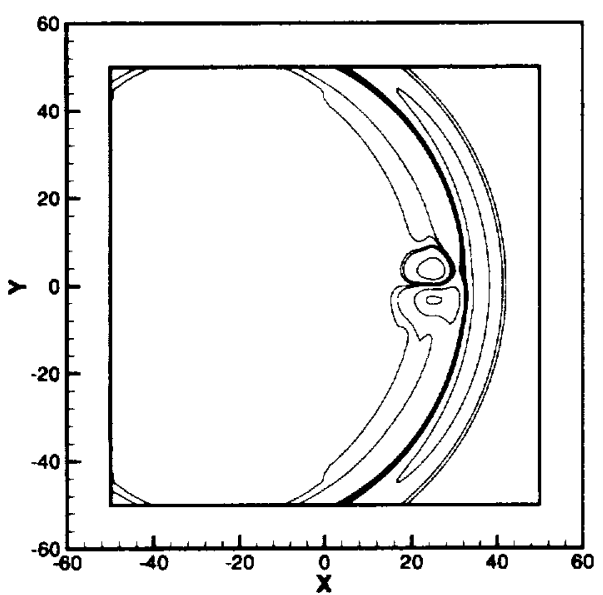

b)

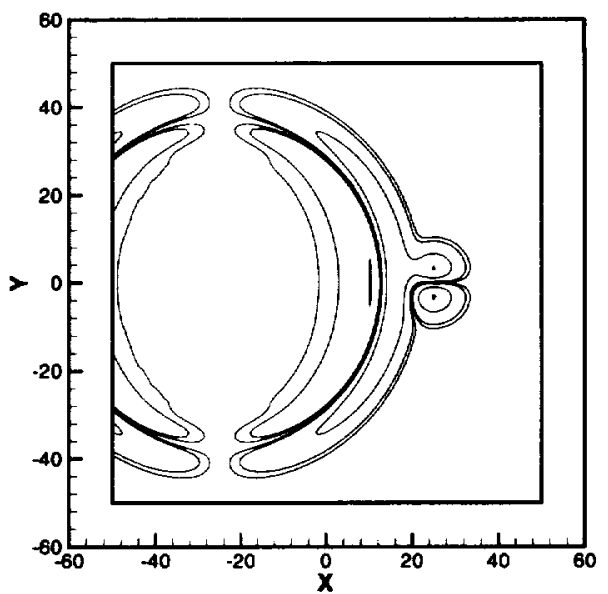

d)

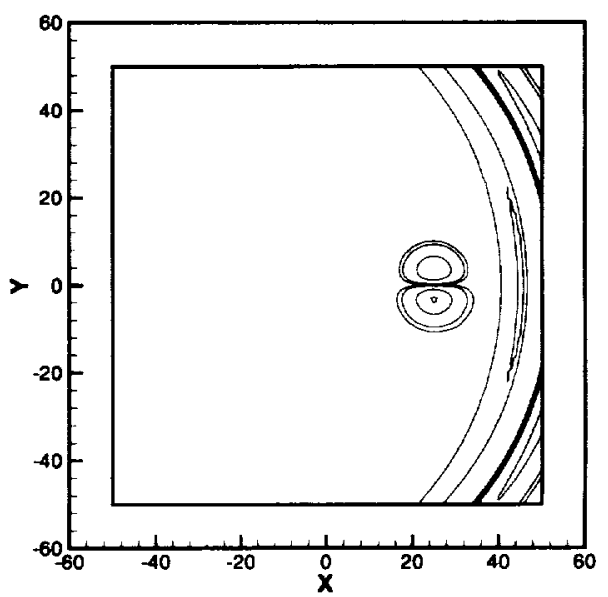

FIG. 2. $u$-velocity contours for a sound pulse, propagating in a quiescent medium. The contour levels are $\pm 0.1, \pm 0.05, \pm 0.01$ and \pm 0.005 with the computed result given at $t=20$ (a), $t=40$ (b), $t=60$ (c) and $t=80$ (d).

more general case of a convecting mean flow.

The first idea that comes to mind is to introduce a new reference frame, moving with a speed of $M$ along $x$ and then apply the PML scheme developed in Sec. 4.1. This approach, however, has the unfortunate consequence that the layer interface becomes a moving interface in physical space.

In [23] the use of a Lorentz-like transformation, connecting the convecting and quiescent wave-equations, is suggested in order to transform the quiescent PML method, such as to be useful in the convecting case. While this approach turns out to work well for the sound-waves, the resulting PML method has an abruptly changing convective velocity for the entropy and vorticity waves, resulting in significant reflection from such waves becoming quiescent exactly at the layer-interface. Moreover, the correct use of this approach in the corner regions of the PML layers is much less clear.

Here we shall take a different approach although we shall rely on the PML schemes developed in the previous section combined with a few other techniques. Introducing layers in which the flow is accelerated into a supersonic region, thereby eliminating the need for absorbing boundary conditions, was recently proposed 


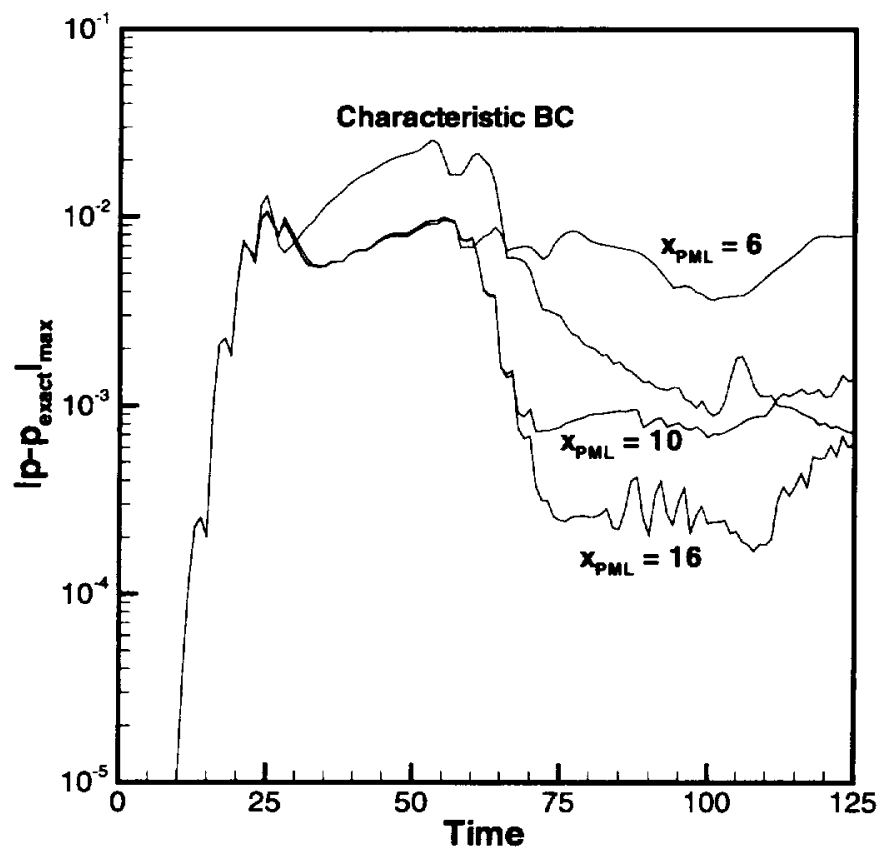

FIG. 3. Maximum error at $x=-48$ as a function of time as computed with different types of boundary conditions and varying width of the PML layer.

in [7] and modified in [9]. While this approach is appealing, it has an undesirable effect on the time-step of the whole computation and primitive sponge layers are still needed to yield an acceptable performance [9].

We propose to decelerate the flow, rather than accelerating it, to approach a quiescent flow inside the layer and then combine this approach with the PML scheme developed in Sec. 4.1. While such a scheme cannot be expected to be perfectly absorbing in the case of a finite layer, it does have the potential of a very efficient absorption, provided the deceleration is sufficiently smooth and the layer width is chosen accordingly.

We propose to consider a PML-like scheme for the convecting case, Eqs.(1)-(2), of the form

$$
\begin{aligned}
& \frac{\partial \rho}{\partial t}+M[1-m(x)] \frac{\partial \rho}{\partial x}=-\frac{\partial u}{\partial x}-\frac{\partial v}{\partial y}-\varepsilon^{\prime} Q_{x}-\mu^{\prime} Q_{y}-\sigma M \rho \\
& \frac{\partial u}{\partial t}+M[1-m(x)] \frac{\partial u}{\partial x}=-\frac{\partial p}{\partial x}-2 \varepsilon u-\varepsilon P_{x} \\
& \frac{\partial v}{\partial t}+M[1-m(x)] \frac{\partial v}{\partial x}=-\frac{\partial p}{\partial y}-2 \mu v-\mu P_{y}-\sigma M v \\
& \frac{\partial p}{\partial t}+M[1-m(x)] \frac{\partial p}{\partial x}=-\frac{\partial u}{\partial x}-\frac{\partial v}{\partial y}-\varepsilon^{\prime} Q_{x}-\mu^{\prime} Q_{y} \\
& \frac{\partial P_{x}}{\partial t}=\varepsilon u, \frac{\partial Q_{x}}{\partial t}=-\varepsilon Q_{x}+u \\
& \frac{\partial P_{y}}{\partial t}=\mu v, \frac{\partial Q_{y}}{\partial t}=-\mu Q_{y}+v .
\end{aligned}
$$

Here $\epsilon$ and $\mu$ remains unchanged from Sec. 4.1. We have introduced $m(x)$, which provides the decelerating 
term by being $m(a) \simeq 0$, with $x=a$ signifies the layer interface, while $m\left(a+x_{P M L}\right) \simeq 1$ at the termination of the absorbing layer. We have found that using the error function provides a good compromise between steepness and smoothness such that

$$
m(z)=\frac{1}{2}\left[1+\frac{2}{\sqrt{\pi}} \int_{0}^{\sigma_{m}\left[z-x_{m}\right]} e^{-t^{2}} d t\right],
$$

where $z=(x-a) / x_{P M L}$ and $\sigma_{m}$ and $x_{m}$ controls the steepness and relative position of the profile, respectively. In Eq.(23) we have also introduced simple absorbing terms in the equations for $\rho$ and $v$. Since the quiescent PML scheme only provides perfect absorption for the sound waves, this is meant to provide a simple mechanism for damping of the entropy and vorticity waves inside the layer. The parameters $\sigma(x)$ can be used to control the strength of this sponge layer for $\rho$ and $v$.

A few comments regarding the scheme, Eq.(23), is appropriate. First of all we note that for $M=0$ we recover Eq.(13). Also since only the diagonal entries of A in Eqs. (1)-(2) are altered the well-posedness of the equations of acoustics remains intact. The philosophy here is that as the convective waves are slowed down, they approach the case of the quiescent acoustics for which Eq.(13) was shown to perform well. Moreover, slowly decelerating the waves as they enter the layer has the beneficial consequence that the wave fronts become increasingly normal to the boundary of the layer - much like water waves always being aligned with the beachfront. Hence, applying characteristic boundary conditions for truncating the PML layer can be expected to be efficient.

4.2.1. A Numerical Example. In order to establish the soundness of the arguments that lead to the PML-like scheme given in Eq.(23), we have conducted a number of experiments using the scheme and initial conditions described in Sec. 4.1.1 and taking $M=0.5$ as the convective Mach number of the mean flow.

The decelerating term, Eq.(24) is generally specified by using $\sigma_{m}=5$ and $x_{m}=0.5$, i.e. the profile is centered in the middle of the absorbing layer. We have taken $\sigma(x)=\varepsilon(x)$, although this is by no means a unique choice and alternatives might well yield better performance that reported here.

Since the layer now has multiple functions, i.e. it decelerates the waves while also acting as an absorbing layer, it is expected that, compared to the quiescent case, slightly wider layers should be used to achieve an acceptable accuracy.

In Fig. 4 we show the temporal development of the density for the initial conditions given in Eq.(21) with $\varepsilon$ and $\mu$ being given in Eq.(22) and the parameters chosen as in Sec. 4.1.1. We have taken the width of the layer as $x_{P M L}=y_{P M L}=20$, i.e. 20 computational cells, and $\Delta t=0.5$. The exact solution is given in $[22]$.

As expected, the sound pulse, as well as the entropy pulse, leaves the computational domain with no noticeable reflections from the layer. In Fig. 5 we show the development of the $u$-velocity component, arriving at similar conclusions.

To address, in a more quantitative manner, the accuracy of the proposed scheme as a function of the width of the layer we have computed the maximum error in the pressure along the line $x=48$ as a function of time. In Fig. 6 we plot the results for increasing width of the layer and compare to the accuracy obtained when using only a characteristic boundary condition to terminated the computational domain.

Indeed we find that using a layer of only 10 cells yields an overall accuracy of the order of the approximation error of the scheme and is superior to that obtained using characteristic boundary conditions only. By increasing the width of the layer to 20 cells, we observe a significant reduction, much like the case of the true PML in Fig. 3, of the reflections from the layer. 
a)

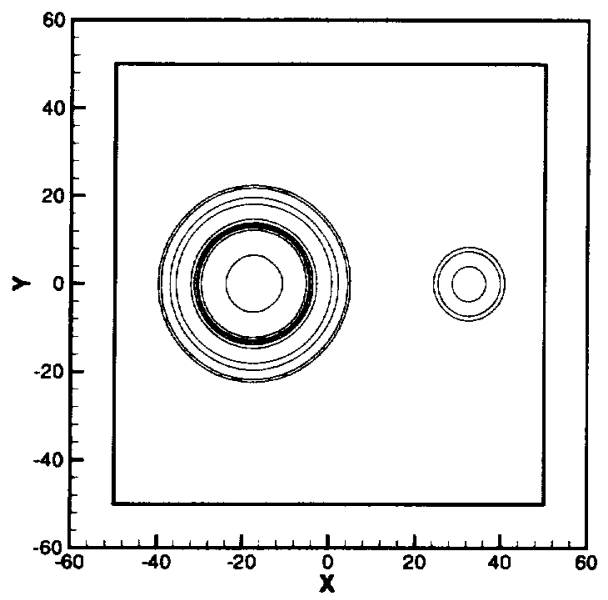

c)

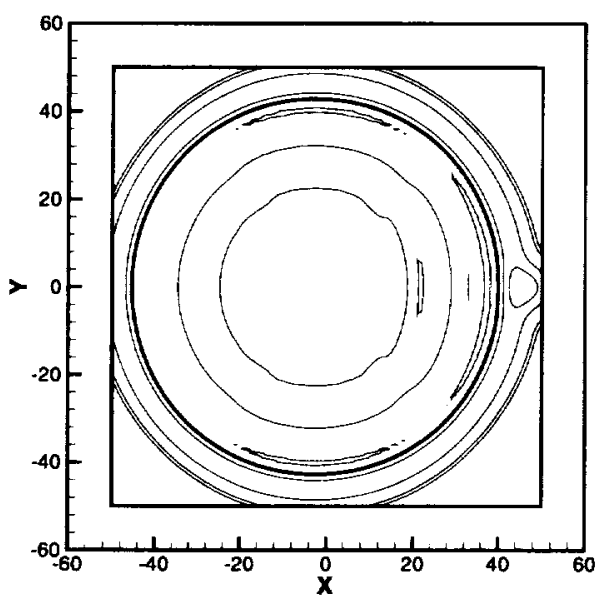

b)

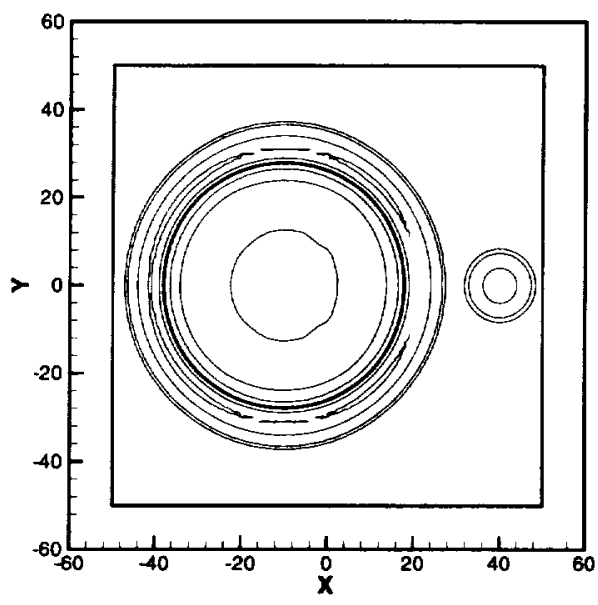

d)

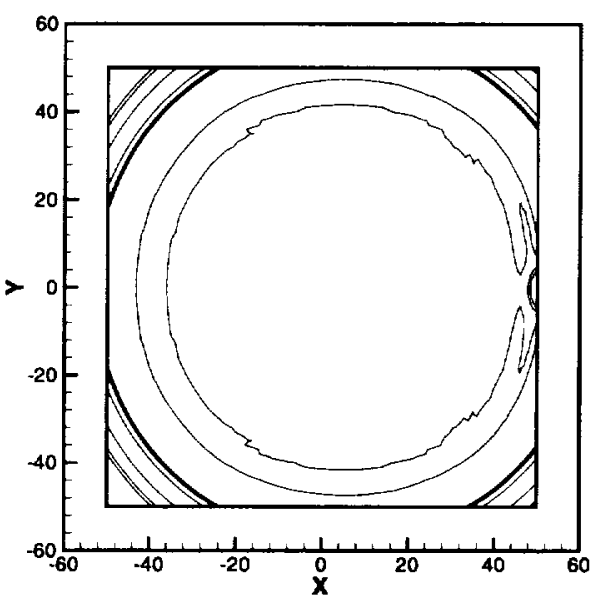

FIG. 4. Density contours for a sound and entropy pulse, propagating in a convecting medium with $M=0.5$. The contour levels are $\pm 0.1, \pm 0.05, \pm 0.01$ and \pm 0.005 with the computed result given at $t=15$ (a), $t=30(b), t=45$ (c) and $t=60$ (d).

As expected, a slightly wider layer, as compared to the results in Sec. 4.1.1, is required in order to obtain an acceptable accuracy. However, rather than increasing the number of cells in the layer one could use a mapping, thereby stretching the grid, combined with a filter inside the layer. This approach was proposed in [6] for the case of acoustics and successfully used for the case of electromagnetics in $[15,16]$. While this approach, certainly, will improve on the performance of the scheme with only little extra computational effort, we have chosen not to implement this technique in order to emphasize that the present schemes do not require the use of a filter in order to maintain stability.

5. Concluding Remarks. The purpose of this paper has been two-fold. In the first part of the paper we provide an analysis of a recently proposed PML method for the equations of acoustics [18]. As remarked in $[18,19]$ these PML methods suffer from intrinsic numerical instabilities and we provided an explanation for this in terms of loss of well-posedness of the split set of equations and, as a result of this, the appearance of ill-posedness under small arbitrary perturbations. Such perturbations will inevitably exist in any numerical implementation of the split set of equations, rendering the schemes inherently unstable unless some kind of 
a)

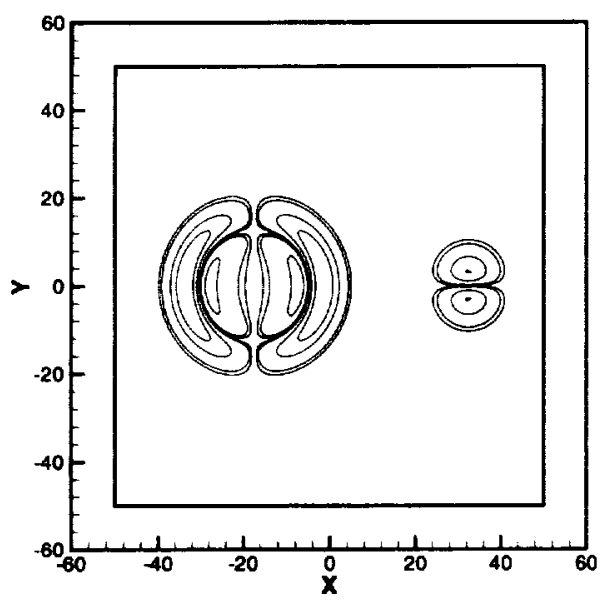

c)

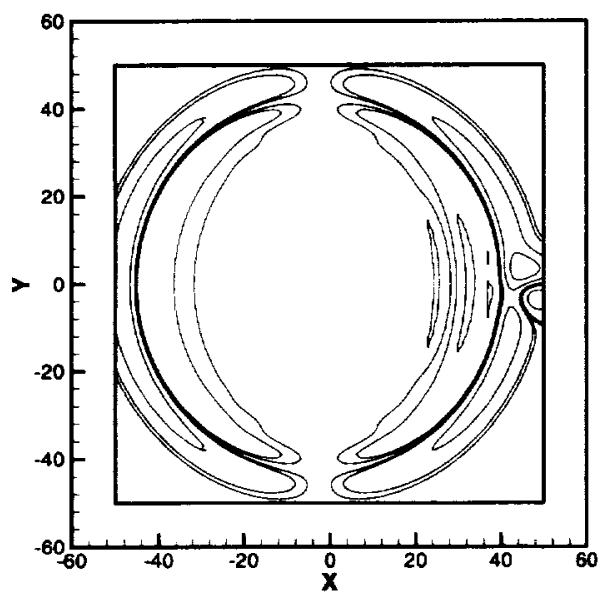

b)

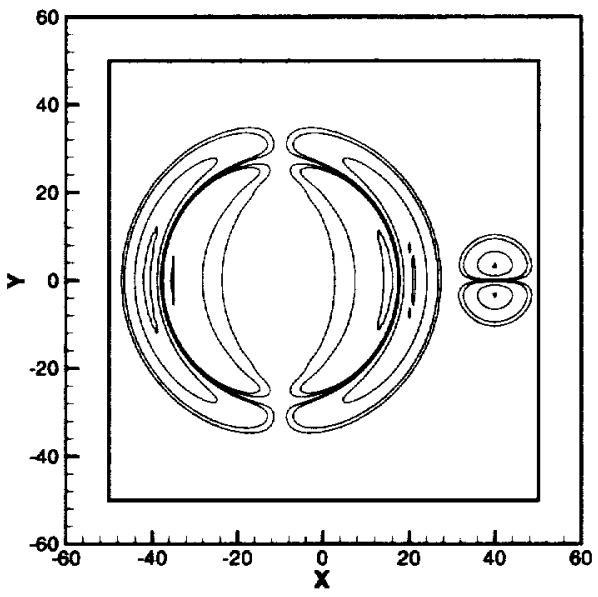

d)

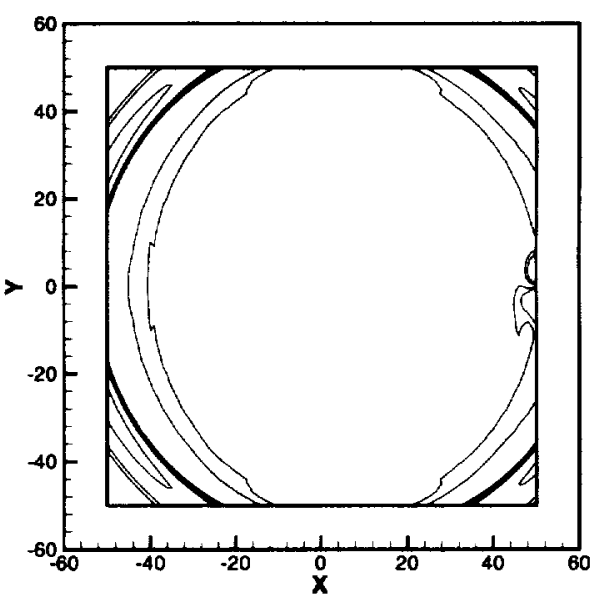

FIG. 5, u-velocity contours for a sound and vorticity pulse, propagating in a convecting medium with $M=0.5$. The contour levels are $\pm 0.1, \pm 0.05, \pm 0.01$ and \pm 0.005 with the computed result given at $t=15(a), t=30(b), t=45$ (c) and $t=60(d)$.

high-frequency damping, e.g. in the form of a low-pass filter, is introduced.

The use of filters is a subject of some controversy. We believe, however, that while there might be numerous physical arguments for applying filters in various situations, it is somewhat of a concern if the numerical scheme, rather than the physics, dictates the need for a filter as is the case of the PML methods in [18]. Indeed, in situations where smooth initial conditions and only smooth boundaries are considered it is troublesome if the solution of a linear hyperbolic system requires the use of filters.

In the second part of the paper we present a PML scheme for the quiescent equations of acoustics and prove that it is indeed absorbing for all frequencies and angles of incidence while remaining strongly well-posed. The properties of the layer is studied in more detail through numerical tests, confirming the analysis.

In the general case of a convecting mean flow, the situation is no perfectly resolved. While we present an absorbing layer scheme that exhibit PML-like behavior, it is not a PML method, but rather a scheme arrived 


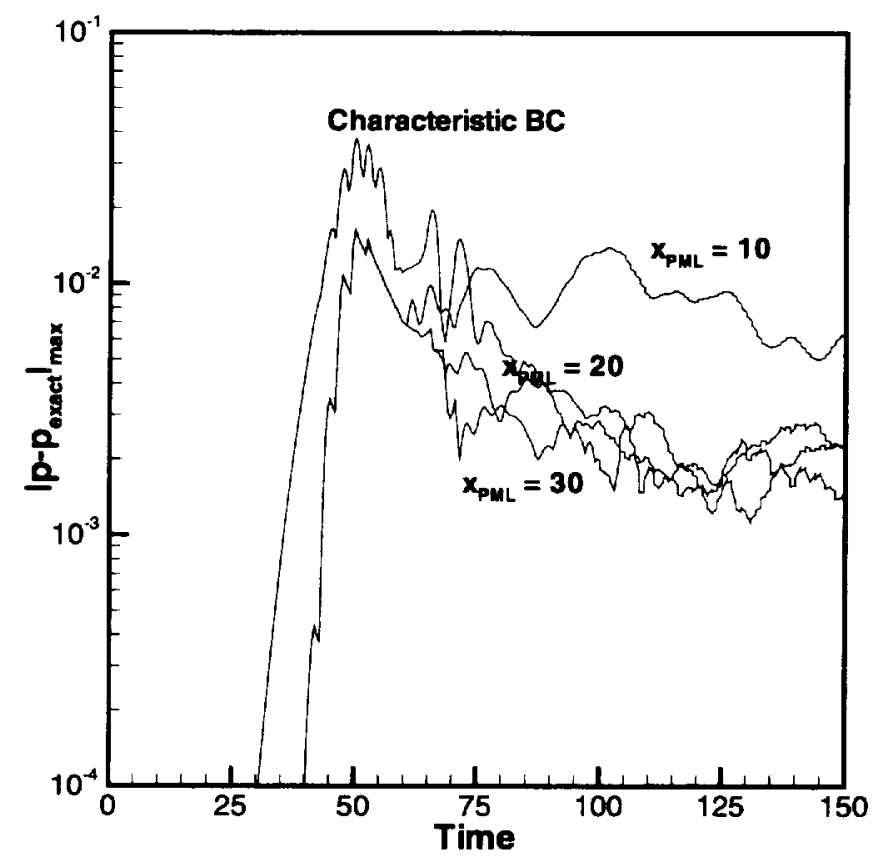

FIG. 6. Maximum error at $x=48$ as a function of time as computed with different types of boundary conditions and varying depth of the absorbing layer.

at by combining several techniques. The scheme remains well-posed and performs well, although it requires the use of a slightly wider layer as compared to the true PML method presented earlier. The advantage of this scheme is that it contains the true PML scheme in the limit of a vanishing mean velocity and extends trivially to the general case of a mean flow which is not aligned with the axis.

The development of a true well-posed PML method for the convective equations of acoustics remains a open challenge and we hope to address this in a future paper.

Acknowledgments. The author would like to express his gratitude to Prof. D. Gottlieb, Brown University, for inspiring and enlightening conversations and Prof. D.A. Kopriva for useful advice. This work was partially supported by DOE grant DE-FG02-95ER25239 and NSF grant ASC-9504002. 


\section{REFERENCES}

[1] K. W. Thompson, Time Dependent Boundary Conditions for Hyperbolic Systems, J. Comput. Phys. 68(1987), pp. 1-24.

[2] B. Engquist AND A. MAJdA, Absorbing Boundary Conditions for the Numerical Simulation of Waves, Math. Comp. 31(1977), pp. 629-651.

[3] R. L. Higdon, Numerical Absorbing Boundary Conditions for the Wave Equation, Math. Comp. 49(1987), pp. 65-90.

[4] A. Bayliss AND E. Turkel, Radiation Boundary Conditions for Wave-Like Equations, Comm. Pure Appl. Math. 33(1980), pp. 707-725.

[5] D. Givoli, Non-Reflecting Boundary Conditions, J. Comput. Phys. 94(1991), pp. 1-29.

[6] T. Colonius, S. K. Lele ANd P. MoIN, Boundary Conditions for Direct Computation of Aerodynamic Sound Generation. AIAA Journal 31(1993), pp. 1574-1582.

[7] S. TA'ASAN AND D. M. NARK, An Absorbing Buffer Zone Technique for Acoustic Wave Propagation. AIAA Paper 95-0146, 1995.

[8] K. Mazaheri and P. L. RoE, Numerical Wave Propagation and Steady-State Solutions: Soft Wall and Outer Boundary Conditions, AIAA Journal 35(1997), pp. 965-975.

[9] J. B. Freund, Proposed Inflow/Outflow Boundary Conditions for Direct Computation of Aerodynamic Sound, AIAA Journal 35(1997), pp. 740-742.

[10] J.-P. Berenger, A Perfectly Matched Layer for the Absorption of Electromagnetic Waves, J. Comput. Phys. 114(1994), pp. 185-200.

[11] S. Abarbanel and D. Gottlieb, A Mathematical Analysis of the PML Method, J. Comput. Phys. 134(1997), pp. 357-363.

[12] L. Zhao AND A. C. Cangellaris, A General Approach for the Development of Unsplit-Field TimeDomain Implementations of Perfectly Matched Layers for FD-TD Grid Truncation, IEEE Microwave Guided Wave Lett. 6(1996), pp. 209-211.

[13] R. W. Zıolkowski, Time-Derivative Lorentz-Material Model Based Absorbing Boundary Conditions, IEEE Trans. Anten. Prop. 1997 - to appear.

[14] P. Petropoulos, L. Zhao and A. C. Cangellaris, A Reflectionless Sponge Layer Absorbing Boundary Condition for the Solution of Maxwell's Equations with High-Order Staggered Finite Difference Schemes, J. Comput. Phys. 1997 - to appear.

[15] B. Yang, D. Gottlieb And J.S. Hesthaven, Spectral Simulations of Electromagnetic Wave Scattering, J. Comput. Phys. 134(1997), pp. 216-230.

[16] B. YAng, D. Gottlieb And J.S. Hesthaven On the Use of PML ABC's in Spectral Time-Domain Simulations of Electromagnetic Scattering, in Proc. of ACES 13'th Annual Review of Progress in Applied Computational Electromagnetics, Monterey, 1997. pp. 926-933.

[17] S. Abarbanel and D. Gottlieb, On the Construction and Analysis of Absorbing Layers in CEM, in Proc. of ACES 13'th Annual Review of Progress in Applied Computational Electromagnetics, Monterey, 1997. pp. 876-883.

[18] F.Q. HU, On Absorbing Boundary Conditions for Linearized Euler Equations by a Perfectly Matched Layer, J. Comput. Phys. 129(1996), pp. 201-219.

[19] J. W. Goodrich and T. Hagstrom, A Comparison of Two Accurate Boundary Treatments for Computational Aeroacoustics, AIAA Paper 97-1585, 1997. 
[20] B. Gustavsson, H.O. Kreiss and J. Oliger, Time Dependent Problems and Difference Methods. John Wiley and Sons, Inc. New York, 1995.

[21] S. Abarbanel and D. Gottlieb, Optimal Time Splitting Methods for the Navier-Stokes Equations and Two- and Three Space Variables, J. Comput. Phys. 41(1981), pp. 1-33.

[22] J. C. HARdin, J. R. Ristorcelli And C. K. W. TAM(Eds.), ICASE/LaRC Workshop on Benchmark Problems in Computational Aeroacoustics (CAA). NASA CP 3300, NASA Langley Research Center, 1995.

[23] E. Turkel and I. Harari, Absorbing Boundary Layers for Wave-Like Equations, Appl. Numer. Math., 1997 - submitted. 


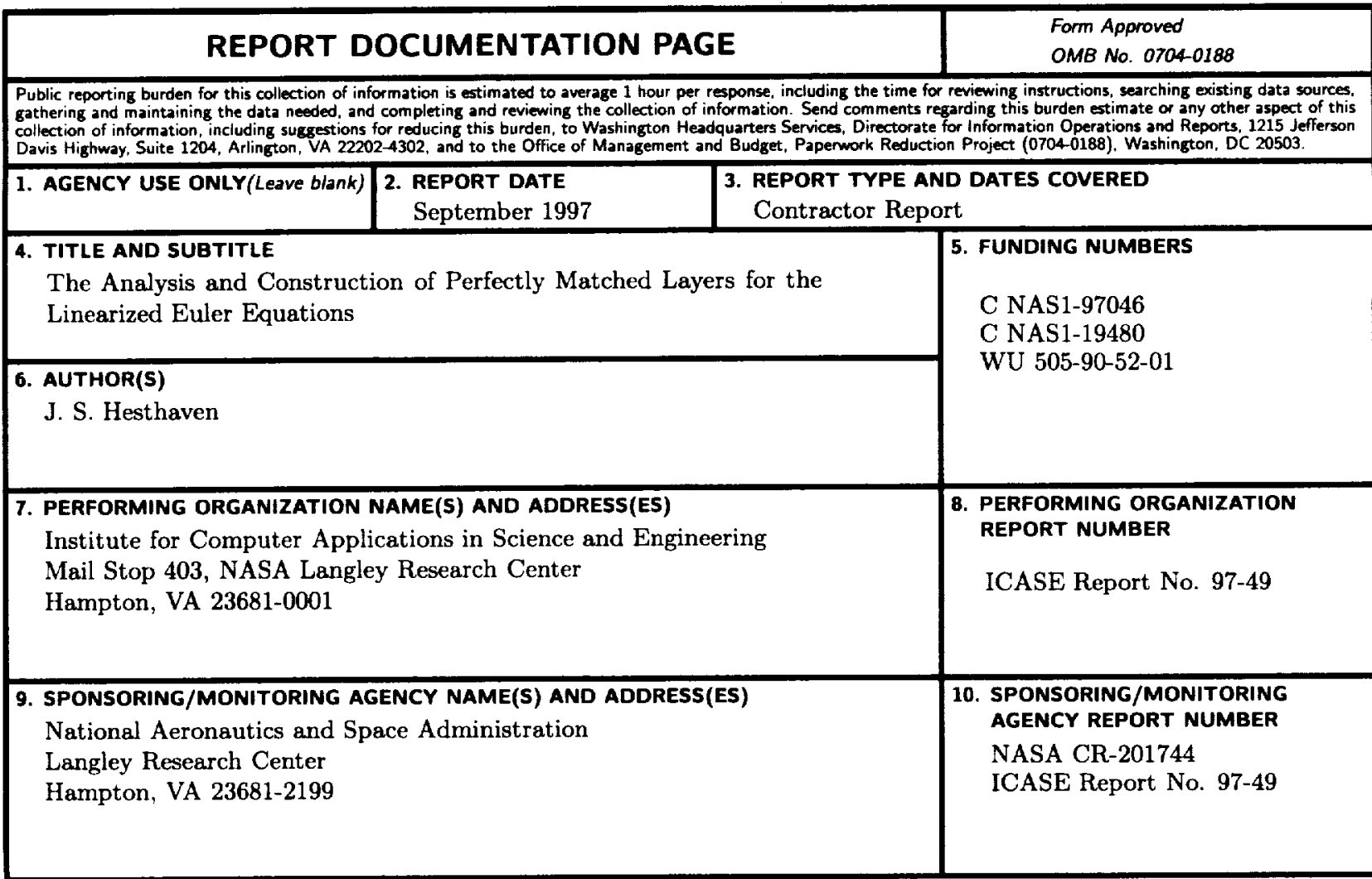

\section{SUPPLEMENTARY NOTES}

Langley Technical Monitor: Dennis M. Bushnell

Final Report

Submitted to Journal of Computational Physics

12a. DISTRIBUTION/AVAILABILITY STATEMENT

12b. DISTRIBUTION CODE

Unclassified Unlimited

Subject Category 34, 64

Distribution: Nonstandard

Availability: NASA-CASI (301)621-0390

13. ABSTRACT (Maximum 200 words)

We present a detailed analysis of a recently proposed perfectly matched layer (PML) method for the absorption of acoustic waves. The split set of equations is shown to be only weakly well-posed, and ill-posed under small low order perturbations. This analysis provides the explanation for the stability problems associated with the spilt field formulation and illustrates why applying a filter has a stabilizing effect.

Utilizing recent results obtained within the context of electromagnetics, we develop strongly well-posed absorbing layers for the linearized Euler equations. The schemes are shown to be perfectly absorbing independent of frequency and angle of incidence of the wave in the case of a non-convecting mean flow. In the general case of a convecting mean flow, a number of techniques is combined to obtain a absorbing layers exhibiting PML-like behavior. The efficacy of the proposed absorbing layers is illustrated though computation of benchmark problems in aero-acoustics.

14. SUBJECT TERMS

acoustics, absorbic boundary conditions, hyperbolic systems

15. NUMBER OF PAGES

\begin{tabular}{|c|c|c|c|}
\hline & & & $\mathrm{A03}$ \\
\hline $\begin{array}{l}\text { 17. SECURITY CLASSIFICATION } \\
\text { OF REPORT } \\
\text { Unclassified }\end{array}$ & $\begin{array}{l}\text { 18. SECURITY CLASSIFICATION } \\
\text { OF THIS PAGE } \\
\text { Unclassified }\end{array}$ & $\begin{array}{l}\text { 19. SECURITY CLASSIFICATION } \\
\text { OF ABSTRACT }\end{array}$ & $\begin{array}{l}\text { 20. LIMITATION } \\
\text { OF ABSTRACT }\end{array}$ \\
\hline NSN 7540-01-280-5500 & & & $\begin{array}{l}\text { Standard form 298( Rev. 2-69) } \\
\text { Prescribed by ANSI Std. Z39-18 } \\
298-102\end{array}$ \\
\hline
\end{tabular}

